\title{
Applications of Modified Adaptive Affine Projection Predictor for Global Positioning System Interference Suppression
}

\author{
Wei-Lung Mao* and Chung-Wen Hung \\ Graduate School of Engineering Science \& Technology and Department of Electrical Engineering, \\ National Yunlin University of Science and Technology, \\ 123 University Road, Section 3, Douliou, Yunlin 64002, Taiwan, R.O.C.
}

(Received March 1, 2017; accepted March 5, 2018)

Keywords: affine projection algorithm (APA), dynamic selection method, global positioning system (GPS) sensor, interference suppression, variable-step size

The global positioning system (GPS) sensor has had a considerable impact on almost all positioning, navigation, timing, and monitoring applications. It provides spread spectrum satellite signals that can be processed in a GPS receiver, and it allows the receiver to estimate information about position, velocity, and time. A low-power GPS signal is susceptible to interference, which can seriously degrade receiver acquisition and tracking performance. Two kinds of modified affine projection filters, namely, variable step-size and dynamic selection structures, are presented for GPS jamming suppression applications. The variable step-size method based on the minimization of the mean square deviation is employed to achieve a higher convergence rate and a lower misadjustment error. The dynamic selection method can update the input vector by selecting a subset component and reduce computational complexity while offering a higher convergence speed. Suppression performance is evaluated via extensive simulation by computing the mean-squared prediction error (MSPE) and signal-to-noise ratio (SNR) improvements. Simulations show that our proposed methods can provide better performance than the conventional affine projection algorithm (APA) structures when severe interference-to-noise ratios (INRs) are experienced.

\section{Introduction}

A global positioning system (GPS) sensor ${ }^{(1-3)}$ employing a direct sequence spread spectrum (DSSS) technique can provide accurate positioning and timing information in a wide range of applications. The reliability and availability of GPS signals have become crucial issues for the fast-growing use of GPS receivers. GPS spreads the bandwidth of transmitted signals with a coarse/acquisition (C/A) code, which results in an approximately $43 \mathrm{~dB}$ processing gain. Thus, the DSSS scheme inherently provides a modest anti-jamming property that can deal with narrowband interference. If a higher jamming power arises, it is necessary to supplement the innate processing gain by using additional signal processing techniques such as adaptive filtering. ${ }^{(4-7)}$

*Corresponding author: e-mail: wlmao@yuntech.edu.tw http://dx.doi.org/10.18494/SAM.2018.1873 
The study of adaptive filters used in anti-jamming has attracted considerable attention in recent years. ${ }^{(6-8,18,19)}$ The most popular adaptive filters are least mean squares (LMS)based algorithms owing to their inherent simplicity. However, LMS-based methods have slow convergence rates and are not suitable for applications affected by nonstationary and impulse interference. Ozeki and Umeda ${ }^{(9)}$ first used an affine subspace projection structure to derive the basic form of affine projection algorithms (APAs). These methods ${ }^{(7,8,10,11)}$ provide the simplicity of structure and fast computation nature of LMS algorithms and reduce gradient noise by exploiting the use of the most recent inputs to improve performance. The APA is a useful family of adaptive filters that offer numerous applications in system identification, acoustic echo cancellation, and impulse interference prevention. ${ }^{(13-16)}$ The family of APAs updates the weight coefficients by multiple, most recent input vectors instead of a single, current data vector applied in the normalized LMS (NLMS) algorithms. In conventional APA methods, the step size is a constant parameter that determines the convergence rate and steady-state excess mean square error. To meet these conflicting requirements, several schemes for controlling the step size have been proposed. ${ }^{(12-14)}$ The method in Ref. 11 resulted in an optimal step size adjusted with the largest decrease in mean square deviation (MSD). These jamming obstacles can be inherently stationary/nonstationary and also be associated with higher order statistics, so variable step-size filters may be more suitable for signal prediction. Recently, various algorithms that concentrate on the number of projection orders have also been proposed. ${ }^{(9,13,15,16)}$ A higher projection order leads to a higher convergence speed, but a larger estimation error can result. The selection of projection order using the MSD criterion may be employed to yield a low computational complexity and a small steady error. The transform domain approach $^{(19)}$ is also proposed for jamming suppression. A reference signal is generated with a wavelet-packettransform-based adaptive predictor, such that the interfering signal can be subtracted from the received signal. This method can mitigate chirp and single-tone interferences with a high interference-to-signal ratio (INR).

In this paper, the performance of the APA for stationary and nonstationary interference cancellation in GPS sensors is considered. The APAs offer an intermediate complexity between the LMS and recursive least squares (RLS) approaches. To improve the tracking capability of APA, two schemes are proposed, i.e., (a) variable step-size APA (VS-APA), and (b) dynamic selection APA (DS-APA) structures. The adaptive step size for APA obtained by minimizing the MSD is utilized to provide higher convergence rates and lower misadjustment errors for interfering signal estimation. To reduce the computational complexity in receiver design, an APA with dynamic selection input vectors is established to improve the convergence rate. Once the prediction of the interfering signal is obtained, an error signal can be computed by subtracting the estimate from the received signal. The error signal is then fed into the correlator for dispreading.

The remainder of this paper is organized as follows. In Sect. 2, the GPS received signal model is described. In Sect. 3, we present the conventional APA, the VS-APA method, and the DS-APA method. In Sect. 4, simulation results and analysis are discussed to verify the proposed methods. In Sect. 5, we conclude this paper. 


\section{GPS Received Signal Models}

GPS satellites transmit ranging codes and navigation data at two frequencies: primary L1 and secondary L2, and only the L1 signal, free for civilian use, is analyzed herein. A simplified block diagram of an anti-jamming GPS model is shown in Fig. 1. The received signal $x(t)$ can be modeled as

$$
x(t)=S_{L 1 i}(t)+n(t)+j(t)=\sqrt{2 P_{t}} d_{i}(t) C_{i}(t) \cos \left(2 \pi f_{L 1} t+\theta\right)+n(t)+j(t),
$$

where $S_{L 1 i}(t)$ is the L1 signal transmitted from $S V_{i}, P_{t}$ is the transmitted signal power, $d_{i}(t)$ is the navigation data bits, $C_{i}(t)$ is the pseudorandom noise spreading sequence (C/A code) with chip duration $T_{c}, f_{L 1}$ and $\theta$ are $\mathrm{L} 1$ carrier frequency $(1575.42 \mathrm{MHz})$ and phase delay, respectively, $n(t)$ is additive white Gaussian noise (AWGN) with variance $\sigma^{2}$, and the jamming source $j(t)$ has a bandwidth much smaller than the GPS spreading bandwidth. Owing to the downconversion step, the spectrum of the signal is shifted to the baseband frequency. To further simplify the analysis, it is assumed that the received signal passes through a filter matched to the chip waveform and is sampled synchronously once during each chip interval. The observation is given as

$$
x(k)=S_{L 1 i}(k)+n(k)+j(k),
$$

where $\left\{S_{L 1 i}(k)\right\},\{n(k)\}$, and $\{j(k)\}$ are discrete time sampled waveforms of $\left\{S_{L 1 i}(t)\right\},\{n(t)\}$, and $\{j(t)\}$, respectively. They are assumed to be mutually independent. The $n(k)$ can be modeled as band-limited and white, and the jamming source being considered has a bandwidth much

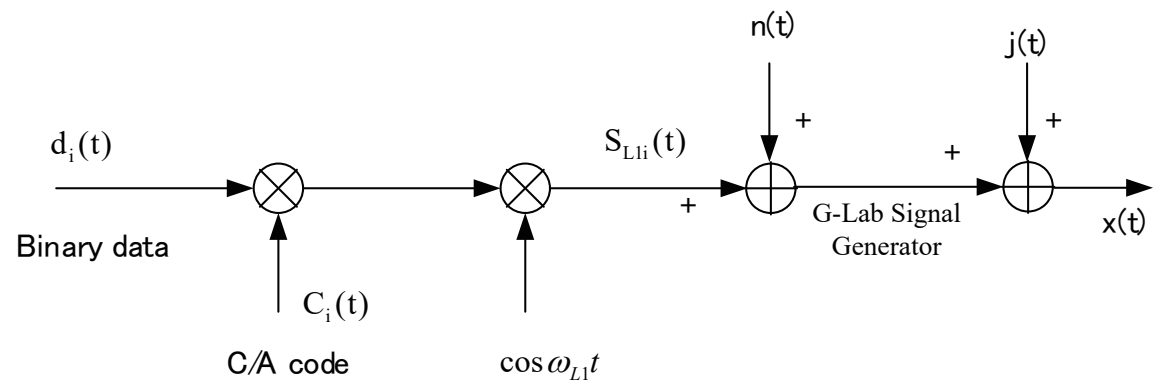

(a)

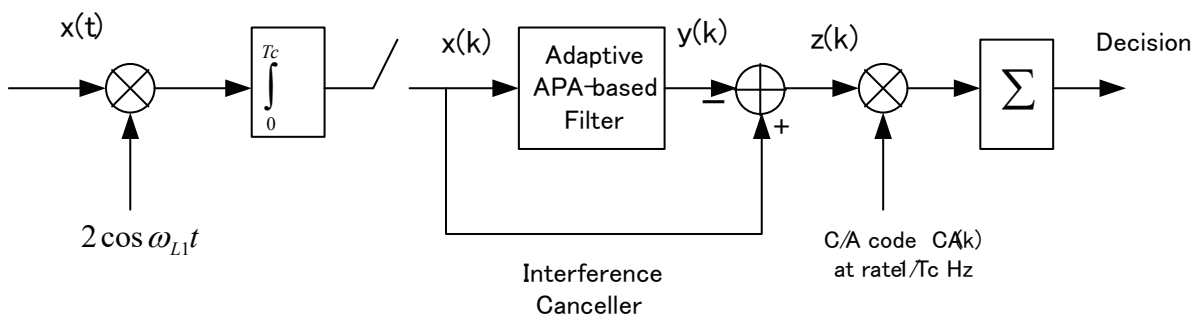

(b)

Fig. 1. GPS spread spectrum system. (a) Transmitter architecture generated by G-Lab software and (b) antijamming receiver. 
narrower than $1 / T_{c}$. The $S_{L 1 i}(k)$ sequence is $d_{i}(k) C_{i}(k)$ for values of \pm 1 . In this paper, four kinds of narrowband interference are considered:

(1) Single-tone continuous wave interference (CWI): $J_{1}(t)=\sqrt{2 P_{J}} \cos \left[\left(\omega_{L 1}+\omega_{\Delta}\right) t+\theta\right]$,

where $P_{J}$ denotes the power, and $\omega_{\Delta}$ is its offset frequency from the central frequency of the spread spectrum signal. The term $\theta$ is the random phase, which is uniformly distributed over the interval $[0,2 \pi)$.

(2) Multi-tone CWI (MCWI): $J_{2}(t)=\sum_{i=1}^{I} \sqrt{2 P_{J i}} \cos \left[\left(\omega_{L 1}+\omega_{\Delta i}\right) t+\theta_{i}\right]$,

where $P_{J i}, \theta_{i}$, and $\omega_{\Delta i}$ represent the power, random phase, and offset frequency of the $i$-th interferer, respectively. The term $I$ is the number of narrowband interferers.

(3) Pulsed CWI (PCWI): $J_{3}(t)=\left\{\begin{array}{cc}A \cos \left(\omega_{L 1}+\omega_{\Delta}\right) t, & (l-1) N_{T} T_{c} \leq t<(l-1) N_{T} T_{s}+N_{1} T_{c} \\ 0, & (l-1) N_{T} T_{c}+N_{1} T_{c} \leq t<l N_{T} T_{c}\end{array}\right.$

where $A$ denotes the amplitude and $T_{c}$ is the sample time. The on-interval period is $N_{1} T_{c}$ seconds long and the off-interval period is $\left(N_{T}-N_{1}\right) T_{c}$ seconds long. The case in which $N_{T}$ and $N_{1}$ are much greater than unity is considered.

(4) Linear FM: $J_{4}(t)=\sqrt{2 P_{J}} \cos \left[\left(\omega_{L 1}+\omega_{\Delta}\right) t+\frac{\alpha_{\Delta}}{2} t^{2}\right]$,

where $P_{J}, \omega_{\Delta}$, and $\alpha_{\Delta}$ represent the power, offset frequency, and frequency rate, respectively. In Fig. 1(b), the narrowband canceller composed of an APA-based filter and a subtractor is employed to suppress the jamming signals. The $\left\{S_{L 1 i}(k)\right\}$ and $\{n(k)\}$ sequences are wideband signals with nearly flat spectra. However, these two sequences cannot be estimated from their past values. The interfering signal $\{j(k)\}$ can be predicted because of its correlated property. The error signal $z(k)$ is

$$
z(k)=S_{L 1 i}(k)+n(k)+j(k)-\hat{j}(k) \cong S_{L 1 i}(k)+n(k),
$$

where $z(k)$ can be obtained as an almost interference-free signal, and the intermediate frequency (IF) component can be demodulated to a navigation signal after code correlation.

\section{Proposed APAs}

While a NLMS method updates the weights based only on the current input vector, the APA can update the weights based on the current and previous input vectors. It is a useful family of adaptive filters in numerous digital signal processing applications. 


\subsection{APA}

An APA includes a family of configurable algorithms designed to improve the performance of other adaptive algorithms, mainly LMS-based ones, especially when input data is highly correlated. The objective of an APA is to minimize this cost function:

$$
\min \left\{\frac{1}{2}\|\boldsymbol{w}(k+1)-\boldsymbol{w}(k)\|^{2}\right\}
$$

subject to: $\boldsymbol{d}(k)-\boldsymbol{X}^{\mathrm{T}}(k) \boldsymbol{w}(k+1)=\mathbf{0}$,

with

$$
\begin{gathered}
\boldsymbol{X}(k)=\left[\begin{array}{llll}
\boldsymbol{x}(k) & \boldsymbol{x}(k-1) & \cdots & \boldsymbol{x}(k-L)
\end{array}\right], \\
\boldsymbol{x}(k)=\left[\begin{array}{llll}
x(k) & x(k-1) & \cdots & x(k-N)
\end{array}\right]^{\mathrm{T}}, \\
\boldsymbol{d}(k)=\left[\begin{array}{llll}
d(k) & d(k-1) & \cdots & d(k-L)
\end{array}\right]^{\mathrm{T}}, \\
\boldsymbol{y}(k)=\boldsymbol{X}^{\mathrm{T}}(k) \boldsymbol{w}(k), \\
\boldsymbol{e}(k)=\boldsymbol{d}(k)-\boldsymbol{y}(k),
\end{gathered}
$$

where $\boldsymbol{X}(k)$ is the input signal matrix, $\boldsymbol{x}(k)$ is the column vector in $\boldsymbol{X}(k), \boldsymbol{d}(k)$ is the desired signal vector, $\boldsymbol{y}(k)$ is the adaptive-filter output, and $\mathrm{L}$ is the data reuse factor. The Lagrange multiplier method is used to turn the constrained minimization into an unstrained one. The unconstrained function to be minimized becomes

$$
\boldsymbol{J}(k)=\frac{1}{2}\|\boldsymbol{w}(k+1)-\boldsymbol{w}(k)\|^{2}+\lambda^{\mathrm{T}}(k)\left[\boldsymbol{d}(k)-\boldsymbol{X}^{\mathrm{T}}(k) \boldsymbol{w}(k+1)\right],
$$

where $\lambda(k)$ is an $1 \times(L+1)$ vector of Lagrange multipliers. The gradient of $\boldsymbol{J}(k)$ with respect to $\boldsymbol{w}(k+1)$ can be represented as

$$
\frac{\partial \boldsymbol{J}(k)}{\partial \boldsymbol{w}(k+1)}=\boldsymbol{w}(k+1)-\boldsymbol{w}(k)-\boldsymbol{X}(k) \lambda(k)
$$

The gradient function above is set to zero, and the weighting vector can be

$$
\boldsymbol{w}(k+1)=\boldsymbol{w}(k)+X(k) \lambda(k)
$$

After substituting Eq. (11) into the constraint relation of Eq. (8) followed by some manipulations, the update equation is

$$
\boldsymbol{w}(k+1)=\boldsymbol{w}(k)+\boldsymbol{X}(k)\left(\boldsymbol{X}^{\mathrm{T}}(k) \boldsymbol{X}(k)\right)^{-1} \boldsymbol{e}(k) .
$$

The APA is represented as 
Initialization: $\boldsymbol{w}(0)=\left[\begin{array}{llll}0 & 0 & \cdots & 0\end{array}\right], 0<\mu \leq 2$

For $k \in\{1, \ldots, \infty\}$,

$$
\begin{gathered}
\boldsymbol{e}(k)=\boldsymbol{d}(k)-\boldsymbol{X}^{\mathrm{T}}(k) \boldsymbol{w}(k), \\
\boldsymbol{w}(k+1)=\boldsymbol{w}(k)+\mu \boldsymbol{X}(k)\left(\boldsymbol{X}^{\mathrm{T}}(k) \boldsymbol{X}(k)+\gamma \boldsymbol{I}\right)^{-1} \boldsymbol{e}(k),
\end{gathered}
$$

where $\mu$ is the learning rate, and $\gamma$ is a small constant added to the matrix $\boldsymbol{X}^{\mathrm{T}}(k) \boldsymbol{X}(k)$ to avoid numerical problems in the matrix inversion.

\subsection{VS-APA}

The conventional APA method uses a constant step size in the coefficient update equation that controls the stability as well as convergence rate and misadjustment. In this method, the algorithm misadjustment decreased as the step-size parameter decreased, and this leads to lower convergence speeds. The APA based on minimizing the mean squared norm of the coefficient error vector is employed. ${ }^{(10)}$ The parameter $\mu$ is chosen such that $\Delta(\mu)$ is maximized, and this choice provides for the MSD to have the largest decrease at each iteration. Let $\tilde{\boldsymbol{w}}(k)$ be a weight error vector, $\tilde{\boldsymbol{w}}(k)=\boldsymbol{w}^{o}-\boldsymbol{w}(k)$, such that

$$
\tilde{\boldsymbol{w}}(k)=\tilde{\boldsymbol{w}}(k-1)-\mu \boldsymbol{X}(k)\left(\boldsymbol{X}^{\mathrm{T}}(k) \boldsymbol{X}(k)\right)^{-1} \boldsymbol{e}(k),
$$

where $\boldsymbol{w}^{o}$ is an unknown column vector to be estimated. Squaring both sides and taking expectations, the MSD can be represented as

$$
E\|\tilde{\boldsymbol{w}}(k)\|^{2}=E\|\tilde{\boldsymbol{w}}(k-1)\|^{2}-\Delta(\mu),
$$

with $\Delta(\mu)=2 \mu \operatorname{Re}\left\{E\left[\boldsymbol{e}^{\mathrm{T}}(k)\left(\boldsymbol{X}^{\mathrm{T}}(k) \boldsymbol{X}(k)\right)^{-1} \boldsymbol{X}^{\mathrm{T}}(k) \tilde{\boldsymbol{w}}(k-1)\right]\right\}-\mu^{2} E\left[\boldsymbol{e}^{\mathrm{T}}(k)\left(\boldsymbol{X}^{\mathrm{T}}(k) \boldsymbol{X}(k)\right)^{-1} \boldsymbol{e}(k)\right]$.

Assume that the noise sequence is identically and independently distributed and statistically independent of the regression data $\boldsymbol{X}(k)$ and neglect the dependence of $\tilde{\boldsymbol{w}}(k-1)$ on past noise. This can lead to the optimum step-size parameter, which can be approximated as

$$
\mu_{0}(k)=\frac{\operatorname{Re}\left\{E\left[\boldsymbol{e}^{\mathrm{T}}(k)\left(\boldsymbol{X}^{\mathrm{T}}(k) \boldsymbol{X}(k)\right)^{-1} \boldsymbol{X}^{\mathrm{T}}(k) \tilde{\boldsymbol{w}}(k-1)\right]\right\}}{E\left[\boldsymbol{e}^{\mathrm{T}}(k)\left(\boldsymbol{X}^{\mathrm{T}}(k) \boldsymbol{X}(k)\right)^{-1} \boldsymbol{e}(k)\right]}
$$




$$
\cong \frac{E\|\boldsymbol{p}(k)\|^{2}}{E\|\boldsymbol{p}(k)\|^{2}+\sigma_{v}^{2} \operatorname{Tr}\left[E\left(\boldsymbol{X}^{\mathrm{T}}(k) \boldsymbol{X}(k)\right)^{-1}\right]},
$$

with $\boldsymbol{p}(k)=\boldsymbol{X}(k)\left(\boldsymbol{X}^{\mathrm{T}}(k) \boldsymbol{X}(k)\right)^{-1} \boldsymbol{X}^{\mathrm{T}}(k) \tilde{\boldsymbol{w}}(k-1)$,

where $\boldsymbol{p}(k)$ is the projection vector of $\tilde{\boldsymbol{w}}(k-1)$ onto $R(\boldsymbol{X}(k))$, the range space of $\boldsymbol{X}(k)$. The projection vector $\boldsymbol{p}(k)$ can be estimated using the time average form. It can be represented as

$$
\hat{\boldsymbol{p}}(k)=\alpha \boldsymbol{p}(k-1)+(1-\alpha) \boldsymbol{X}(k)\left(\boldsymbol{X}^{\mathrm{T}}(k) \boldsymbol{X}(k)\right)^{-1} \boldsymbol{e}(k),
$$

where $\alpha$ is a smoothing factor in the range of $[0,1)$. The vector $\boldsymbol{p}(k)$ is substituted by $\hat{\boldsymbol{p}}(k)$. The VS-APA becomes

$$
\text { Initialization: } \hat{\boldsymbol{w}}(0)=\left[\begin{array}{llll}
0 & 0 & \ldots & 0
\end{array}\right]^{\mathrm{T}} \text {. }
$$

For $k \in\{1, \ldots, \infty\}$,

$$
\begin{gathered}
\boldsymbol{w}(k)=\boldsymbol{w}(k-1)+\mu_{0}(k) \boldsymbol{X}(k)\left(\boldsymbol{X}^{\mathrm{T}}(k) \boldsymbol{X}(k)\right)^{-1} \boldsymbol{e}(k), \\
\mu_{0}(k)=\mu_{\max } \frac{\|\hat{\boldsymbol{p}}(k)\|^{2}}{\|\hat{\boldsymbol{p}}(k)\|^{2}+C}, \\
\hat{\boldsymbol{p}}(k)=\alpha \hat{\boldsymbol{p}}(k-1)+(1-\alpha) \boldsymbol{X}(k)\left(\boldsymbol{X}^{\mathrm{T}}(k) \boldsymbol{X}(k)\right)^{-1} \boldsymbol{e}(k),
\end{gathered}
$$

where $\mu_{\max }$ is chosen in the range of $[0,2)$ to guarantee the stability of the algorithm, and $C$ is a constant that is related to the noise disturbance variance $\sigma_{v}^{2} \operatorname{Tr}\left[E\left(\boldsymbol{X}^{\mathrm{T}}(k) \boldsymbol{X}(k)\right)^{-1}\right]$.

\subsection{DS-APA}

Adaptive APAs with dynamic selection update belong to the family of adaptive methods that update only a portion of filter parameters at each iteration. These architectures can reduce computational complexity while attempting to maintain close performance to their full update structure. In this section, the dynamic selection of a subset of input vectors is presented to improve the convergence performance of the APA. Let $\tilde{\boldsymbol{w}}(k)$ be a weight error vector, $\tilde{\boldsymbol{w}}(k)=\boldsymbol{w}^{o}-\boldsymbol{w}(k)$, such that

$$
\tilde{\boldsymbol{w}}(k)=\tilde{\boldsymbol{w}}(k-1)-\mu \boldsymbol{X}(k)\left(\boldsymbol{X}^{\mathrm{T}}(k) \boldsymbol{X}(k)\right)^{-1} \boldsymbol{e}(k),
$$


where $\boldsymbol{w}^{o}$ is an unknown column vector to be estimated. Squaring both sides and taking expectations, the MSD becomes

$$
E\|\tilde{\boldsymbol{w}}(k)\|^{2}=E\|\tilde{\boldsymbol{w}}(k-1)\|^{2}-\Delta,
$$

with $\Delta=2 \mu \operatorname{Re}\left\{E\left[\boldsymbol{e}^{\mathrm{T}}(k)\left(\boldsymbol{X}^{\mathrm{T}}(k) \boldsymbol{X}(k)\right)^{-1} \boldsymbol{X}^{\mathrm{T}}(k) \tilde{\boldsymbol{w}}(k-1)\right]\right\}-\mu^{2} E\left[\boldsymbol{e}^{\mathrm{T}}(k)\left(\boldsymbol{X}^{\mathrm{T}}(k) \boldsymbol{X}(k)\right)^{-1} \boldsymbol{e}(k)\right]$, where $\Delta$ is a function of the input vectors. The function $\Delta$ is maximized for iteration $k$, and the biggest decrease can be obtained in MSD from iteration $(k-1)$ to iteration $k$. Since it is not feasible to know the exact expected value, the instantaneous value is replaced in the equation. It can be approximated as

$$
\begin{aligned}
& \bar{\Delta}=\mu(2-\mu) \boldsymbol{e}^{\mathrm{T}}(k)\left(\boldsymbol{X}^{\mathrm{T}}(k) \boldsymbol{X}(k)\right)^{-1} \boldsymbol{e}(k)-2 \mu \sigma_{v}^{2} \operatorname{Tr}\left[\left(\boldsymbol{X}^{\mathrm{T}}(k) \boldsymbol{X}(k)\right)^{-1}\right]^{-1} \\
& \approx \mu(2-\mu)\left\{\left(\frac{\boldsymbol{e}_{0}^{2}(k)-2 \sigma_{v}^{2}(2-\mu)^{-1}}{\|\boldsymbol{x}(k)\|^{2}}\right)+\left(\frac{\boldsymbol{e}_{1}^{2}(k)-2 \sigma_{v}^{2}(2-\mu)^{-1}}{\|\boldsymbol{x}(k-1)\|^{2}}\right)+\cdots+\left(\frac{\boldsymbol{e}_{L-1}^{2}(k)-2 \sigma_{v}^{2}(2-\mu)^{-1}}{\|\boldsymbol{x}(k-L)\|^{2}}\right)\right\},
\end{aligned}
$$

with $\boldsymbol{e}_{n}(k)=\boldsymbol{d}(k-n)-\boldsymbol{x}(k-n) \boldsymbol{w}(k-1),(n=0,1, \ldots, L)$,

where $\sigma_{v}^{2}$ is the measurement noise power, and $\operatorname{Tr}[\bullet]$ means the matrix trace. Assuming that the diagonal elements of $\boldsymbol{X}^{\mathrm{T}}(k) \boldsymbol{X}(k)$ are much larger than the off-diagonal ones, the off-diagonal components are ignored to simplify the computation burden in each iteration. If $\boldsymbol{e}_{n}^{2}(k)$ is larger than $2 \sigma_{v}^{2}(2-\mu)^{-1}, \boldsymbol{x}(k-n)$ can maximize $\bar{\Delta}$. The weight update needs to be carried out with the input vector satisfying $\boldsymbol{e}_{n}^{2}(k)>2 \sigma_{v}^{2}(2-\mu)^{-1}$ at every iteration for the largest MSD reduction. The update equation can be expressed as

$$
\text { Initialization: } \boldsymbol{w}(0)=\left[\begin{array}{llll}
0 & 0 & \cdots & 0
\end{array}\right]
$$

For $k \in\{1, \ldots, \infty\}$,

$$
\boldsymbol{w}(k)=\left\{\begin{array}{cc}
\boldsymbol{w}(k-1), & \boldsymbol{K}(k)=0 \\
\boldsymbol{w}(k-1)+\mu \boldsymbol{X}_{S_{\boldsymbol{K}(k)}}(k)\left(\boldsymbol{X}_{S_{\boldsymbol{K}(k)}^{\mathrm{T}}}^{\mathrm{T}}(k) \boldsymbol{X}_{S_{\boldsymbol{K}(k)}}(k)\right)^{-1} \boldsymbol{e}_{S_{\boldsymbol{K}(k)}}(k), & \text { otherwise }
\end{array}\right.
$$

with $\boldsymbol{X}_{S_{\boldsymbol{K}(k)}}(k)=\left[\begin{array}{llll}\boldsymbol{x}\left(k-t_{1}\right) & \boldsymbol{x}\left(k-t_{2}\right) & \cdots & \boldsymbol{x}\left(k-t_{\boldsymbol{K}(k)}\right)\end{array}\right]^{\mathrm{T}}$,

$\boldsymbol{e}_{S_{\boldsymbol{K}(k)}}(k)=\left[\begin{array}{llll}\boldsymbol{e}_{t_{1}}(k) & \boldsymbol{e}_{t_{2}}(k) & \cdots & \boldsymbol{e}_{t_{\boldsymbol{K}(k)}}(k)\end{array}\right]^{\mathrm{T}}$.

The input vectors are selected when each input vector satisfies the following criterion: 


$$
\left|\boldsymbol{e}_{S_{i}}(k)\right|>\boldsymbol{e}_{t h}, \boldsymbol{e}_{t h}>m \sqrt{2 \sigma_{v}^{2}(2-\mu)^{-1}}, i=1,2, \ldots, \boldsymbol{K}(k), 0<\boldsymbol{K}(k)<L,
$$

where $m$ is the tuning parameter, and $\sigma_{v}^{2}$ is the noise power, which can be estimated from the GPS signal power and thermal noise power. From the received signal model, it is assumed that $S_{L 1 i}(k), j(k)$, and $n(k)$ are statistically independent, and the filter outputs $y_{s}(k), y_{n}(k)$, and $y_{j}(k)$ are also statistically independent. At the affine projection filter outputs, the disturbance due to noise and signal components can be expressed as the sum of $y_{s}(k)$ and $y_{n}(k)$. The output signal of the DS-APA filter can be represented as

$$
y(k)=\boldsymbol{w}^{\mathrm{T}}(k) \boldsymbol{x}(k)=\boldsymbol{w}^{\mathrm{T}}(k)\left[\boldsymbol{s}_{L 1 i}(k)+n(k)+j(k)\right]=y_{s}(k)+y_{n}(k)+y_{j}(k) .
$$

The average power of the disturbance, for a given signal with parameters $\boldsymbol{w}(k)$, can be calculated as

$$
\begin{aligned}
\sigma_{v}^{2}(k) & =E\left[y_{s}^{2}(k)+y_{n}^{2}(k)\right] \\
& =\boldsymbol{w}^{\mathrm{T}}(k) E\left[\boldsymbol{s}_{L 1 i}(k) \boldsymbol{s}_{L 1 i}^{\mathrm{T}}(k)+\boldsymbol{n}(k) \boldsymbol{n}^{\mathrm{T}}(k)\right] \boldsymbol{w}(k) \\
& =\|\boldsymbol{w}(k)\|^{2}\left[E\left[s_{L 1 i}^{2}(k)\right]+\sigma_{n}^{2}(k)\right] .
\end{aligned}
$$

The received signal levels are not expected to exceed $153 \mathrm{dBW}$ for the C/A code components on the L1 channels. ${ }^{(2,3)}$ Typically, the signal power for an SV is from 1 to $5 \mathrm{~dB}$ higher than the minimum specified levels, depending on the elevation angle and SV block. The disturbance bounds of signal level and noise level can be estimated from the GPS signal specification, and the noise level can be utilized in the DS-APA filter.

\section{Simulation Results}

The results of the simulating adaptive APA-based filtering methods are obtained to confirm the jamming rejection characteristics and properties, and our proposed method can adjust the filter effectively to track the interfering signal. Two indices are employed to demonstrate the performances. They are the SNR improvement ratio and mean-squared prediction error (MSPE). The definitions are as follows.

(1) SNR improvement: The metric adopted to verify the steady-state performance is the "SNR improvement," which is defined in Ref. 4 and given by

$$
S N R_{\text {improvement }}=10 \log \left[E\left|x(k)-S_{L 1 i}(k)\right|^{2} / E\left|z(k)-S_{L 1 i}(k)\right|^{2}\right](\mathrm{dB}) .
$$

(2) Average MSPE: The MSPE is used as an index to evaluate the convergence rate of transient responses for various algorithms. It is defined as 


$$
\begin{gathered}
M(k)=\frac{1}{S I M_{\text {num }}}\left(\sum_{i=1}^{S I M_{\text {num }}} e_{i}^{2}(k)\right), \\
M_{M S P E}(n)=\log \left[\frac{1}{100}\left(\sum_{i=\left((n-1)^{*} 100\right)+1}^{n^{* 100}} M(i)\right),\right.
\end{gathered}
$$

where $S I M_{\text {num }}$ is the total number of simulations (which is 500 here), and $e_{i}(k)$ is the predicted error of the $k$-th iteration for the $i$-th run. In this simulation, the received signal is band-passfiltered, amplified, down-converted to IF, and then digitized. The intermediate frequency $\left(f_{I F}\right)$ is fixed at $4.1304 \mathrm{MHz}$, and a sampling frequency $\left(f_{S}\right)$ of $16.36 \mathrm{MHz}$ is selected and binomially distributed with a value $d_{i}(k)$ of \pm 1 , and $C_{i}(k)$ is randomly selected with uniform probability from 24 PRN codes of GPS. The G-Lab tool ${ }^{(17)}$ is used to generate the GPS signal. The noise power can be approximated using thermal noise $\left(=K T_{E} B\right)$, where $K$ is Boltzmann's constant $(1.3806 \mathrm{E}-23$ $\left.\mathrm{JK}^{-1}\right), B$ is the bandwidth in $\mathrm{Hz}$, and $T_{E}$ is the effective noise temperature in Kelvin. The ambient temperature and equipment noise factors are dominant, and a typical effective noise temperature for a GPS receiver is $513 \mathrm{~K}$. This noise power is set to be $-138.5 \mathrm{dBW}$, ${ }^{(3)}$ and the power of the signal $S_{L 1 i}(k)$ is set to $-158 \mathrm{dBW}$. The simulation results are ensemble-averaged over 100 independent runs, and 1500 data points are obtained in each run.

In this section, we illustrate the results of a computer simulation of an anti-jamming GPS sensor system. Four types of algorithm are compared, namely, NLMS, APA, VS-APA, and DS-APA methods. The following parameters are chosen: (a) NLMS method: The length of the tapped delay filter is set to 32 , the converge rate $\mu_{n}$ is set to 0.01 , and parameter gamma $\tau$ is set to $10^{-11}$. (b) APA method: The tap number of the standard APA filter is 32 , the data reuse parameter $L$ is set to 8 , the convergence factor $\mu_{n}$ is set to 0.01 , and the parameter gamma $\tau$ is set to $4 \times 10^{-11}$. (c) DS-APA method: The tap number is set to 32 , the data reuse factor $L$ is set to 32 , the adaptation constant $\mu$ is 0.01 , and the parameter $m$ is set to 1 . The noise power $\sigma_{v}^{2}$ can be estimated from GPS specifications and set to be $1.5849 \times 10^{-14}(=-138.5 \mathrm{dBW})$, and the threshold $\boldsymbol{e}_{t h}$ is set to $1.2621 \times 10^{-7}$. (d) VS-APA method: The tap number is set to 32 , the data reuse factor is set to 32 , the adaptation constant $\mu_{\max }$ is 0.5 , and the parameter $\alpha$ is set to 0.9 .

The interference suppression performances are described as follows:

(A) Single-tone CWI: Figures 2 and 3 present the SNR improvements and averaged MSPE for single-tone CWI, respectively. The frequency of the interference signal is set to $0.45 \mathrm{MHz}$, and the input INR is varied from 20 to $50 \mathrm{~dB}$. Figure 2 shows that the DS-APA schemes are superior in terms of SNR improvement compared with other filters over a wide range of INR, especially in the case of high interference power. In the steady-state condition, the average SNR improvements of the DS-APA method are 1.39, 3.15, and $8.48 \mathrm{~dB}$ higher than those of the APA, VS-APA, and NLMS, respectively. The VS-APA method can also have a better performance in case of lower interference power. From the Figs. 3(a) to 3(d), the averaged MSPE learning curves are shown for different INR inputs. The VS-APA method has a higher convergence rate when the INRs are in the range of 20 to $40 \mathrm{~dB}$, but it has a lower convergence speed under higher INR conditions. It is obvious that the proposed DS-APA method indeed provides a 


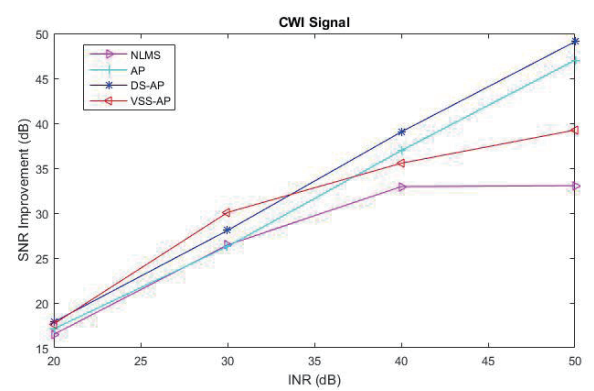

Fig. 2. (Color online) Single-tone CWI suppression performances of SNR improvement vs INR.

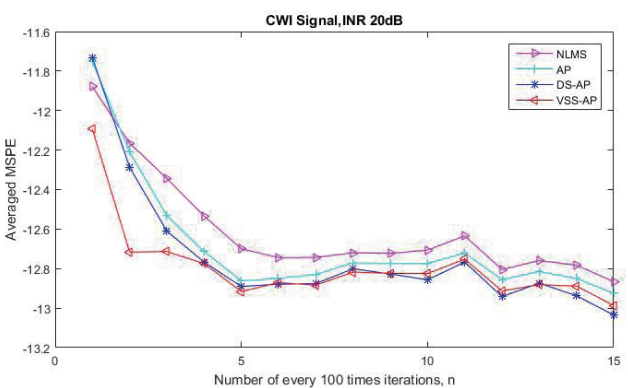

(a)

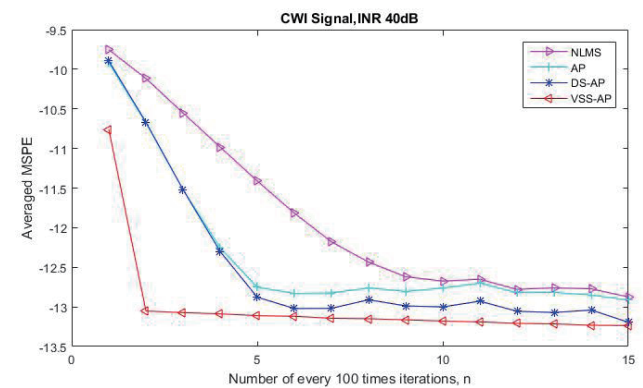

(c)

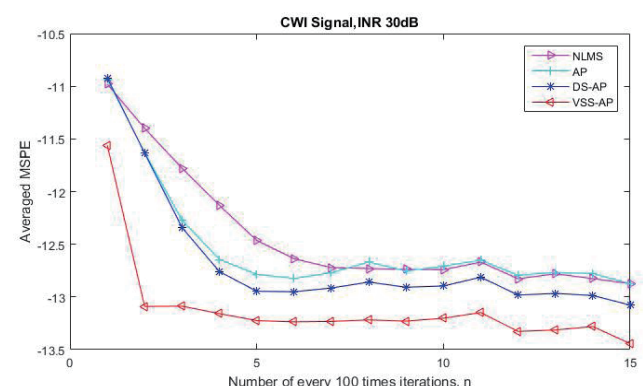

(b)

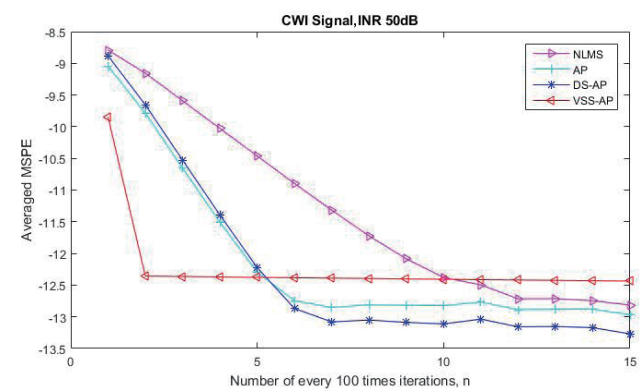

(d)

Fig. 3. (Color online) Single-tone CWI suppression performances of averaged MSPE vs number of every 100 iterations $n$ for (a) $I N R=20 \mathrm{~dB}$, (b) $I N R=30 \mathrm{~dB}$, (c) $I N R=40 \mathrm{~dB}$, and (d) $I N R=50 \mathrm{~dB}$.

better transient response and a shorter convergence time than the other methods. The NLMS is the worst in the convergence comparisons. The DS-APA method can decline significantly to $-13 \mathrm{~dB}$ in 500 iterations, while the other methods reach the steady state slowly and have larger MSPE values.

(B) MCWI: The multi-tone test results are shown in Figs. 4 and 5. The interfering signal considered is a four-tone signal, and the offset frequencies are randomly generated in the range of $f_{I F} \pm 1.023 \mathrm{MHz}$. The four offset frequencies are kept constant at $f_{\Delta i}=-0.68,0.555,0.63$, and $1.02 \mathrm{MHz}$, and $\theta_{i}$ 's are i.i.d. random phases uniformly distributed over the interval $[0,2 \pi)$. The unknown multi-tone interference, which is commonly encountered in spread spectrum systems, provides a stronger variation than the single-tone CWI. Both DS-APA and VS-APA methods, which can vary the number of input vectors and step-size parameters, are always adapted to 


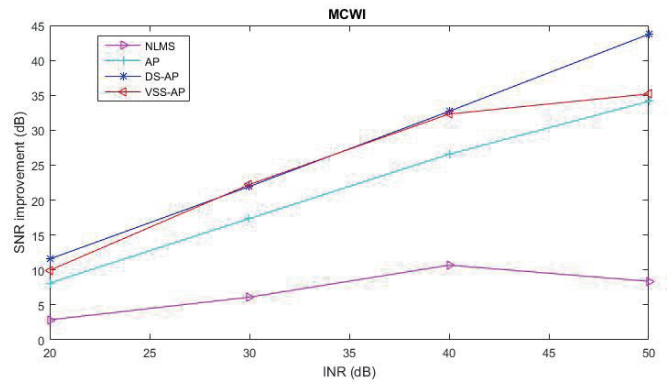

Fig. 4. (Color online) MCWI suppression performances of SNR improvement vs INR.

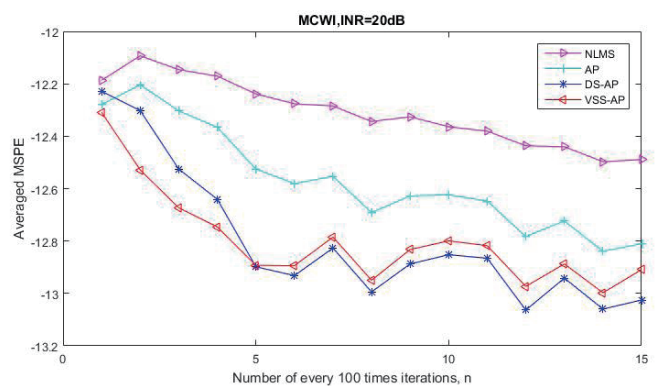

(a)

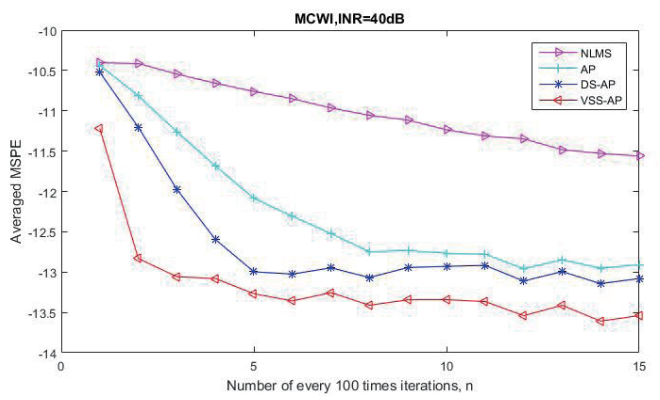

(c)

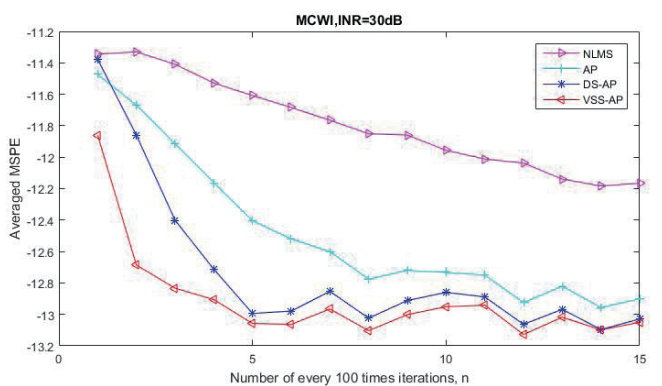

(b)

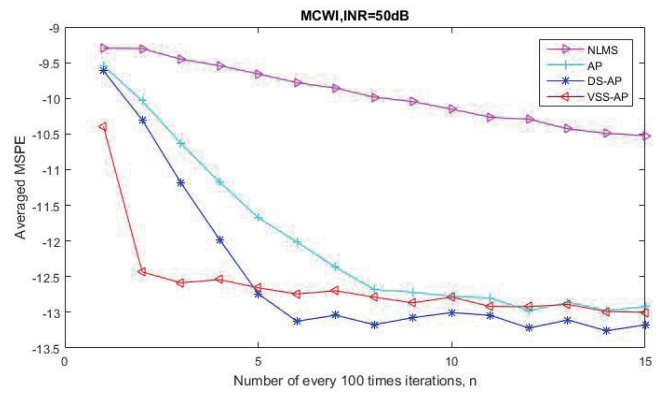

(d)

Fig. 5. (Color online) MCWI suppression performances of averaged MSPE vs number of every 100 iterations $n$ for (a) $I N R=20 \mathrm{~dB}$, (b) $I N R=30 \mathrm{~dB}$, (c) $I N R=40 \mathrm{~dB}$, and (d) $I N R=50 \mathrm{~dB}$.

track the multi-tone with the higher convergence performance in the steady state. On average, the DS-APA algorithm offers 11.34, 2.37, and $19.14 \mathrm{~dB}$ more in terms of SNR improvements than APA, VS-APA, and NLMS methods, respectively. The DS-APA and VS-APA methods can perform with faster convergence than both APA and NLMS structures. The averaged MSPE can be reduced markedly to $-12.8 \mathrm{~dB}$ in 500 iterations, whereas the other approaches reach the steady state after 1200 iterations and offer higher MSPE values.

(C) PCWI: The offset frequency is chosen as $0.2 \mathrm{MHz}$. The on-interval is during the 1 st to the 650th samples, the off-interval is from the 651st to the 1300th iteration points, and the period $N_{T}$ is set at 1300 . A $50 \%$ duty cycle is used because it is reported to be the most damaging setting. Figure 6 illustrates that the DS-APA and VS-APA methods achieve a lower misadjustment behavior in the steady state than the APA and NLMS methods during both the on- and off- 


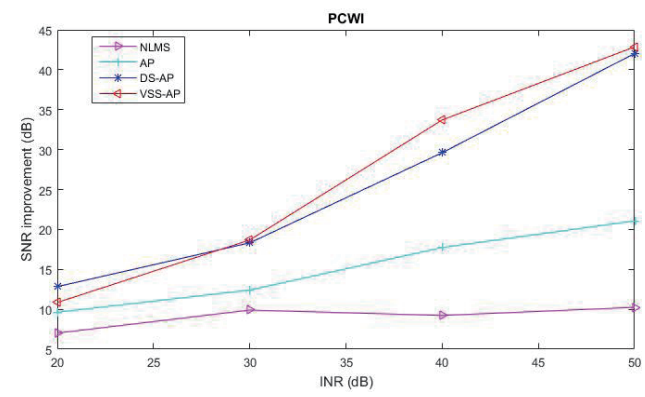

Fig. 6. (Color online) PCWI suppression performances of SNR improvement vs INR.

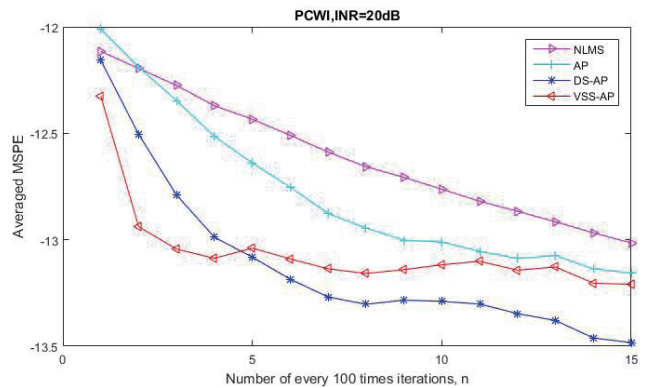

(a)

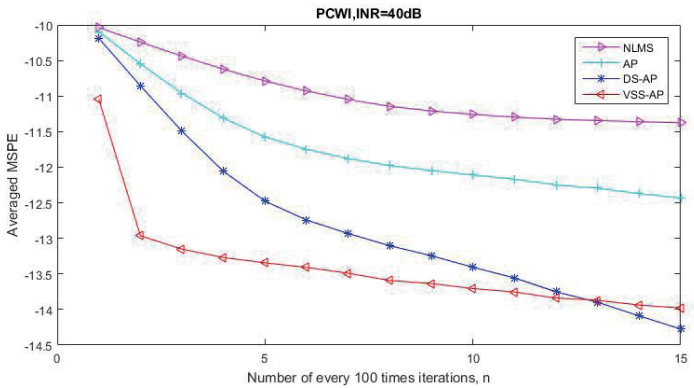

(c)

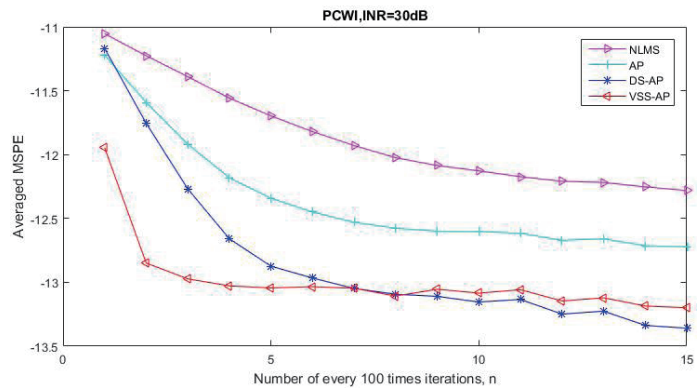

(b)

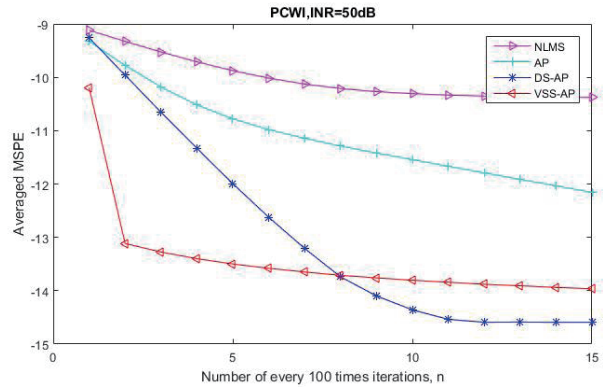

(d)

Fig. 7. (Color online) PCWI suppression performances of averaged MSPE vs number of every 100 iterations $n$ for (a) $I N R=20 \mathrm{~dB}$, (b) $I N R=30 \mathrm{~dB}$, (c) $I N R=40 \mathrm{~dB}$, and (d) $I N R=50 \mathrm{~dB}$.

intervals. The DS-APA method can perform 10.84, 0.16 , and $18.35 \mathrm{~dB}$ more in terms of SNR improvements than the APA, VS-APA, and NLMS methods, respectively. It is shown in Fig. 7 that the DS-APA and VS-APA methods provide better filtering performance and convergence rates than the other methods.

(D) Linear FM: The simulation results of linear FM interference cancellation are shown in Figs. 8 and 9. The starting frequency is set to $4.1304 \mathrm{MHz}$, the sweep bandwidth is $5 \mathrm{KHz}$, and the frequency rate $\alpha_{\Delta}$ is set to 8 . The DS-APA method can vary the number of selected input vectors for the largest MSD reduction and achieve superior performance compared with the other schemes in transient and steady states. On average, the DS-APA method offers 1.30, 4.31, and $12.87 \mathrm{~dB}$ more SNR improvement than the APA, VS-APA, and NLMS methods, respectively. The NLMS method performs poorly at estimating nonstationary interference 


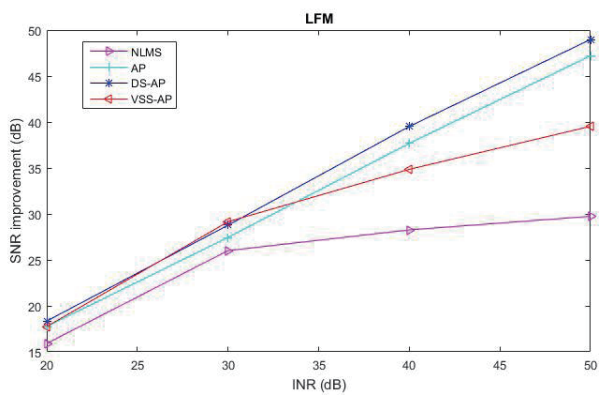

Fig. 8. (Color online) Linear FM suppression performances of SNR improvement vs INR.

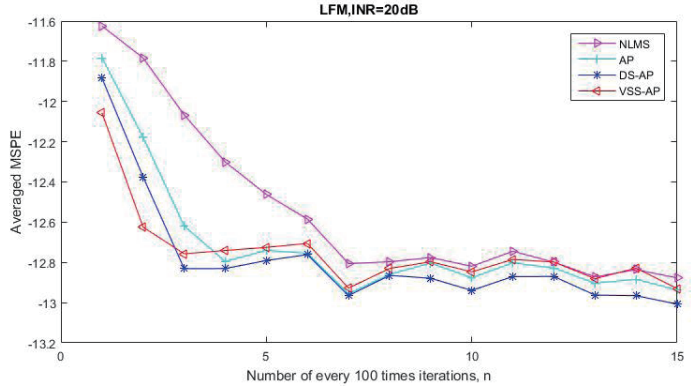

(a)

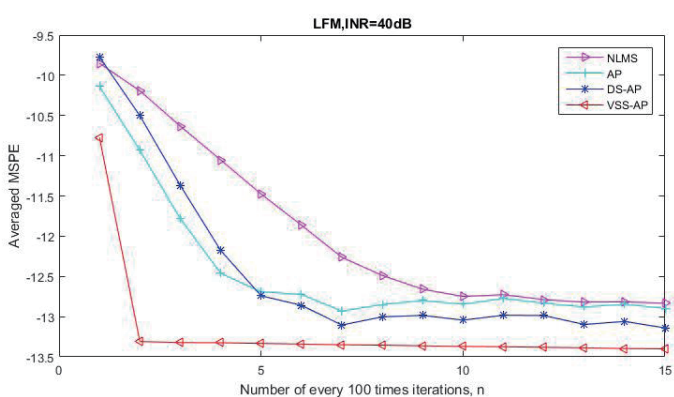

(c)

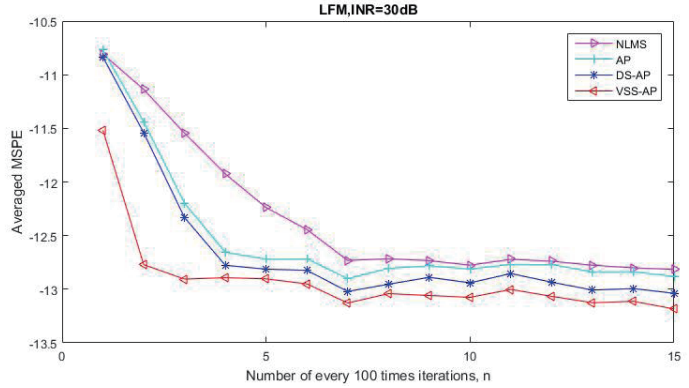

(b)

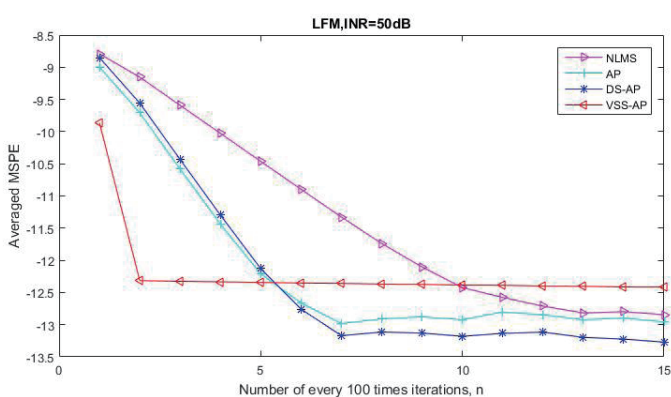

(d)

Fig. 9. (Color online) Linear FM suppression performances of averaged MSPE (dB) vs number of every 100 iterations $\mathrm{n}$ for (a) $I N R=20 \mathrm{~dB}$, (b) $I N R=30 \mathrm{~dB}$, (c) $I N R=40 \mathrm{~dB}$, and (d) $I N R=50 \mathrm{~dB}$.

signals. Because the step size of the NLMS filter is constant, the corresponding convergence rate is very low and limited. The VS-APA method with a variable step size can have a high convergence rate at low INR values, but it has a larger misadjustment error at high INR values.

\section{Conclusions}

In this paper, we present the modified APA-based filtering methods trained with variable step-size and dynamic selection methods for GPS sensor interference cancellation. The variable step-size scheme, which is optimized with respect to the reduction of MSD, is applied in an APA filter to enhance the convergence speed and improve tracking. The DS-APA method also 
uses the MSD criterion to satisfy the characteristics of fast convergence and low computational complexity. The adaptive filtering algorithms are derived and the corresponding interference cancellation performances are presented. On average, the proposed DS-APA and VS-APA filters with powerful learning algorithms can robustly estimate the stationary and nonstationary interfering signals, which are single-tone CWI, MCWI, PCWI, and linear FM. Our proposed adaptive filtering schemes indeed achieve improved SNR and prediction error compared with those of the traditional filters under various INR conditions. The APA-based methods presented herein can be realized into digital signal processing systems by software and become cost-effective implementations.

\section{Acknowledgments}

The authors would like to thank the Ministry of Science and Technology of the Republic of China, Taiwan, for financially supporting this research under Contract No. MOST 105-2622-E224-010 -CC3, MOST 106-2622-E-224-017-CC3, and MOST 105-2218-E-150-004.

\section{References}

1 E. D. Kaplan and C. J. Hegarty: Understanding GPS: Principles and Applications (Artech House, Norwood, MA, 2006).

2 B. Parkinson and J. J Spilker: GPS: Theory and Applications (AIAA, Washington, DC, 1996).

3 M. S. Braasch: Proc. IEEE 87 (1999) 48.

4 L. A. Rusch and H. V. Poor: IEEE Trans. Commun. 42 (1995) 1969.

5 S. Haykin and L. Li: IEEE Trans. Signal Process. 43 (1995) 526.

6 S. G. Glisic, M. Aarne, V. P. Kaasila, and M. K. Pajkovic: IEEE Trans. Commun. 43 (1995) 136.

7 P. S. R. Diniz: Adaptive Filtering: Algorithms and Practical Implementation (Springer, New York, 2008).

8 L. Ping and R. Lu: Int. Conf. Quality, Reliability, Risk Maintenance and Safe Engineering (2013) 2043.

9 K. Ozeki and T. Umeda: Electron. Commun. Jpn. 67 (1984) 19.

10 S. J Kong, K. Y. Hwang, and W. J. Song: IEEE Signal Process. Lett. 14 (2007) 529.

11 H. C. Shin, A. H. Sayed, and W. J. Song: IEEE Signal Process. Lett. 11 (2004) 132.

12 K. Marryas: Digital Signal Process. 20 (2010) 502.

13 J. Shin, J. Yoo, and P. Park: Electron. Lett. 48 (2012) 483.

14 T. Shao, Y. R. Zheng, and J. B. Benesty: IEEE Signal Process. Lett. 17 (2010) 327.

15 S. Wang, J. Eeng, and C. K. Tse: IEEE Trans. Circuit Syst. 60 (2013) 811.

16 M. Shams, E. Abadi, V. Mehrdad, and M. Norouzi: Int. J. Innovative Comput. Info. Control 8 (2012) 1313.

17 C. Dionisio, D. Citterico, and G. Pirazzi: 2010 5th ESA Workshop on Satellite Navigation Technologies and European Workshop on GNSS Signals and Signal Processing (NAVITEC) (2011) 1.

18 Y. R. Chien: IEEE Syst. J. 9 (2015) 451.

19 Y. R. Chien, P. Y. Chen, and S. H. Fang: IEICE Trans. Fundamentals Electron. Commun. Comput. Sci. 100 (2017) 602. 


\section{About the Authors}

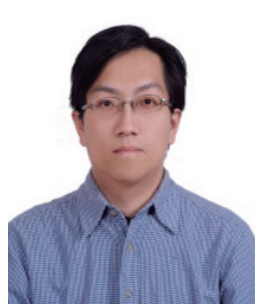

Wei-Lung Mao was born in Taiwan, R.O.C. in 1972. He received his B.S. degree in electrical engineering from National Taiwan University of Science and Technology in 1994, and his M.S. and Ph.D. degrees in electrical engineering from National Taiwan University in 1996 and 2004, respectively. $\mathrm{He}$ is now an associate professor in the Department of Electrical Engineering and Graduate School of Engineering Science and Technology, National Yunlin University of Science and Technology. His research interests are in precision motion control, intelligent and adaptive control systems, satellite navigation systems, adaptive signal processing, neural networks, and communication electronics.

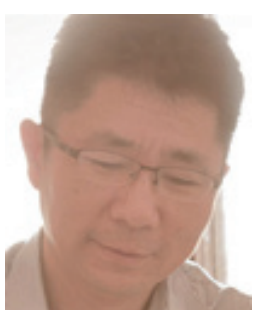

Chung-Wen Hung received his B.S. degree from Feng Chia University, Taiwan, in 1900, and his M.S. and Ph.D. degrees from National Taiwan University, Taiwan, in 2001 and 2007, respectively. From 2009 to 2016, he was an assistant professor at National Yunlin University of Science and Technology, Taiwan. Since 2016, he has been an associate professor at the same university. His research interests are in motor control, Internet of Things (IoT), signal processing, and microcontroller applications. 